FORSCHUNG - AUSBILDUNG - WEITERBILDUNG

Bericht $\mathrm{Nr} .50$

\title{
A STOCHASTIC PARTICLE SYSTEM ASSOCIATED WITH THE \\ SPATIALLY INHOMOGENEOUS BOLTZMANN EQUATION
}

\author{
Wolfgang Wagner
}

UNIVERSITÄT KAISERSLAUTERN

Fachbereich Mathematik

Erwin-Schrödinger-Straße

D - 6750 Kaiserlautern

März 1991 


\title{
A STOCHASTIC PARTICLE SYSTEM ASSOCIATED WITH THE SPATIALLY INHOMOGENEOUS BOLTZMANN EOUATION
}

\author{
Wolfgang Wagner
}

\section{INTRODUCTION}

The efficient numerical treatment of the Boltzmann equation is a very important task in many fields of application (cf., e.g., [Cercignani-1988], [Proceedings-1989], [Gropengiesser et al.-1990]). Most of the practically relevant numerical schemes are based on the simulation of large particle systems that approximate the evolution of the distribution function described by the Boltzmann equation. In particular, stochastic particle systems play an important role in the construction of various numerical algorithms (cf. [Belotserkovskij/Yanitskij -1975], [Nanbu-1983], [Ermakov et al.-1984], [Babovsky-1986], [ I1lner/Neunzert-1987] , [ Ivanov/Rogazinskij-1988]).

The basic approach to the derivation of the Boltzmann equation from a stochastic model is due to M.Kac (cf. [Kac-1956,1959], [McKean-1966,1975]), who considered the spatially homogeneous case. In recent years, there has been a growing interest in the study of more general stochastic models including the spatially inhomogeneous case (cf. [Skorokhod1983], [Kondyurin-1986], [Khisamutdinov-1986], [Arsen'ev-1987], [Lukshin-1989], [Smirnov-1989], [Ivanov/Rogazinskij-1989]). Ivanov and Rogazinskij reported that a stochastic model for the spatially inhomogeneous Boltzmann equation has already been considered in the paper [Leontovich-1935].

The purpose of this paper is to contribute to this field of research. A stochastic particle system is presented that reproduces the Boltzmann equation in the limit as the number of particles goes to infinity. The spatially inhomogeneous 
Boltzmann equation with an external force and rather general boundary conditions is considered. The stochastic model contains parameters allowing to perform asymmetric as well as fictitious collisions between the particles. Thus, some details of the relation between the particle system and the Boltzmann equation become more transparent.

Restricting assumptions have been avoided in this paper whenever it seemed to be possible. Thus, the construction of the stochastic model as well as the transition to the limiting equation contain some heuristic steps. This is the price to be paid for the generality which has been achieved. A review with a comprehensive bibliography concerning rigorous results on the convergence of stochastic particle systems to the Boltzmann equation and a unified derivation of various stochastic particle methods for the numerical treatment of the Boltzmann equation will be provided in separate publications.

This paper is organized as follows. Section 2 contains some general facts concerning Markov processes with jumps.

In Section 3 , the basic Markor process describing the behavior of a large particle system is introduced. This process is constructed on the basis of the free flow process, that defines the motion of a single particle between the collisions, and the collision process, that governs the mechanism of collisions between different particles.

In Section 4, a limiting equation for the empirical measure of the particle system is derived in a heuristic way. The relation of this equation to the Boltzmann equation is studied in Section 5 .

The following notations are used in this paper:

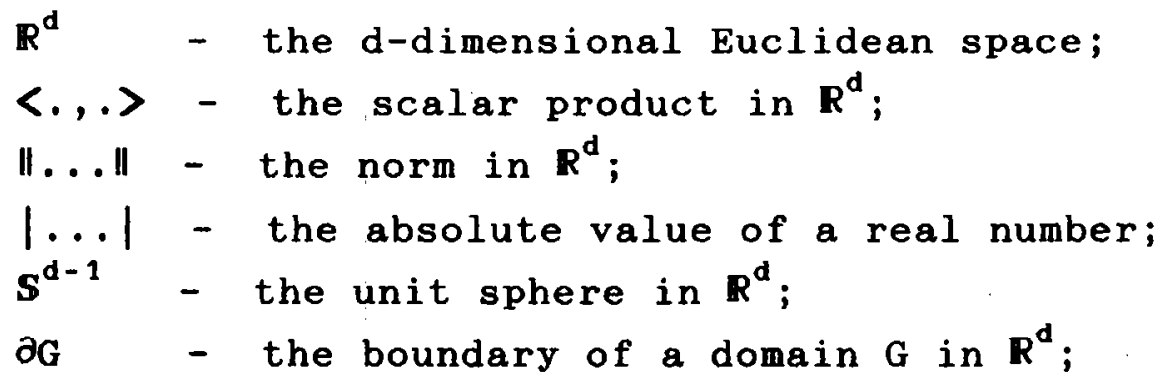


$n(x)$ - the inner normal at the point $x \in \partial G$;

$\delta_{z} \quad-$ the measure concentrated in the point $z \in \mathbb{R}^{d}$;

$\vartheta \quad$ - Dirac's delta-function;

I $\quad$ - the indicator function of a set I;

$\nabla_{z} \quad-$ the vector of the partial derivatives $\left(\frac{\partial}{\partial z_{1}}, \ldots, \frac{\partial}{\partial z}\right) ;$

2. SOME REMARKS CONCERNiNg MARKOV PROCESSES WITH JUMPS

In the first part of this section, we consider a Markov process $z(t), t \geq t_{0}$, with an infinitesimal generator of the form

$$
\mathcal{A}^{(\mathrm{jump})}(\varphi)(\mathrm{z})=\int_{\mathbb{R}^{\mathrm{d}}}[\varphi(\mathrm{y})-\varphi(\mathrm{z})] q(\mathrm{z}, \mathrm{dy})
$$

where $\varphi$ is an arbitrary continuous bounded function on $\mathbb{R}^{d}$. The family of measures $q$ is supposed to be such that

$$
\lambda(z):=q\left(z, \mathbb{R}^{d}\right) \leq \lambda_{\max }<\infty \quad, \quad \forall z \in \mathbb{R}^{d} .
$$

The pathwise behavior of this process is the following ( $c f$. [Ventsel'-1975, p.198] or [Feller-1966, p.389]). The process waits at the state $z\left(t_{0}\right)=z$ a random time $\tau$, which is exponentially distributed with the parameter $\lambda(z)$,

$$
\operatorname{Prob}\{\tau \geq s\}=\exp (-\lambda(z) s), s \geq 0 \text {. }
$$

At the $t$ ime $t=t_{0}+\tau$, the process jumps to a state $y \in \mathbb{R}^{d}$, which is distributed according to the probability measure

$$
\lambda(z)^{-1} q(z, d y) \text {. }
$$

Then, the evolution goes on analogously.

In general, the parameter $\lambda(z)$ of the waiting distribution (2.3) as well as the jump distribution (2.4) depend on the current state $z$.

The measures $q$ appearing in (2.1) are not unique in the sense that the integral does not change if one adds measures of the form $\gamma(z) \delta_{z}(d y), \gamma \geq 0$. Thus, under the assumption $(2.2)$, the 
infinitesimal generator (2.1) can be represented in the form (cf. [Ethier/Kurtz-1986, p.163])

$\mathscr{A}^{(j u m p)}(\varphi)(z)=\int_{\mathbb{R}^{d}}[\varphi(y)-\varphi(z)]\left\{\left(\lambda_{\max }-\lambda(z)\right) \delta_{z}(d y)+q(z, d y)\right\}$,

which suggests the following equivalent description of the pathwise behavior of the process. As before, the process waits an exponentially distributed time $\tau$, but the parameter is now $\lambda_{\max }$ and does not depend on $z$. The jump distribution is

$\left(1-\lambda(z) / \lambda_{\max }\right) \delta_{z}(d y)+\left(\lambda(z) / \lambda_{\max }\right) \lambda(z)^{-1} q(z, d y)$

and depends on $z$. The distribution (2.5) can be interpreted in such a way that, with the probability $\lambda(z) / \lambda_{\max }$, the jump has the distribution $(2.4)$, and, with the probability $1-\lambda(\mathrm{z}) / \lambda_{\max }$, the jump is fictitious.

For our purposes, it is convenient to consider the class of jump processes with

$$
q(z, d y)=\int_{\theta} \delta+T(z, \theta)(d y) Q(z, \theta) \mu(d \theta),
$$

where $\mu$ is a measure on a parameter set $\theta, Q$ is a real-valued function on $\mathbb{R}^{d} \otimes \theta$, and $T$ is a transformation from $\mathbb{R}^{d} \otimes$ into $\mathbb{R}^{d}$.

For this special class of processes, fictitious jumps can be introduced in another way. Suppose

and

$$
Q(z, \theta) \leq Q_{\operatorname{maj}}(z, \theta)
$$

$\lambda_{\mathrm{maj}}(\mathrm{z}):=\int_{\Theta} \mathrm{Q}_{\mathrm{maj}}(\mathrm{z}, \theta) \mu(\mathrm{d} \theta) \leq \mathrm{const}<\infty, \forall \mathrm{z} \in \mathbb{R}^{\mathrm{d}}$.

Then, the infinitesimal generator (2.1) can be transformed in the following way,

$A^{(j u m p)}(\varphi)(z)=\int_{\theta}[\varphi(z+T(z, \theta))-\varphi(z)] Q(z, \theta) \mu(d \theta)$ 


$$
\begin{aligned}
& =\int_{\theta}^{1} \int_{0}^{1}[\varphi(z+T(z, \theta))-\varphi(z)] \mathbb{I}_{I}(z, \theta, \eta) Q_{m a j}(z, \theta) d \eta \mu(d \theta) \\
& =\int_{\theta}^{1} \int_{0}^{1}\left[\varphi\left(z+T(z, \theta) \mathbb{I}_{I}(z, \theta, \eta)\right)-\varphi(z)\right] Q_{m a j}(z, \theta) d \eta \mu(d \theta),
\end{aligned}
$$

where $I:=\left\{(z, \theta, \eta): \eta<Q(z, \theta) / Q_{\text {maj }}(z, \theta)\right\}$.

Consequently, the pathwise behavior of the corresponding process can be described as follows. The parameter of the waiting time distribution $(2.3)$ is $\lambda_{\mathrm{maj}}(z)$ defined in $(2.8)$. The jump distribution is

$$
\lambda_{m a j}(z)^{-1} \int_{\theta}^{1} \int_{0} \delta_{z+T(z, \theta) I_{I}(z, \theta, \eta)}(d y) Q_{m a j}(z, \theta) d \eta \mu(d \theta)
$$

Thus, the jump parameters $\theta$ and $\eta$ are to be generated according to the probability measure

$$
\lambda_{\mathrm{maj}}(z)^{-1} Q_{\mathrm{maj}}(z, \theta) \mu(\mathrm{d} \theta) \mathbb{I}_{[0,1]}(\eta) \mathrm{d} \eta
$$

The new state is calculated as a deterministic transformation of the old state and the jump parameters,

$$
y=z+T(z, \theta) I_{I}(z, \theta, \eta)
$$

This can be interpreted according to the definition of the set $I$ in such a way, that, with the probability $Q(z, \theta) / Q_{\text {maj }}(z, \theta)$, the new state is

$$
y=z+T(z, \theta),
$$

and, with the probability $1-Q(z, \theta) / Q_{m a j}(z, \theta)$, the jump is fictitious.

Note that in the case

$$
Q_{\operatorname{maj}}(z, \theta)=Q_{\max }(\theta)
$$

neither the parameter of the waiting distribution nor the distribution of the jump parameters depend on the current state $z$.

It should be mentioned that the introduction of fictitious 
jumps does not change the process itself, since the infinitesimal generator remains the same. It provides just another way of modeling the process.

In the second part of this section, we consider various superpositions of other Markovian evolutions with the jump mechanism described in the first part. More precisely, we are interested in Markov processes with an infinitesimal generator of the form

$$
A=A^{(0)}+A^{(j u m p)},
$$

defined on an appropriate set of functions $\mathscr{D}(\mathbb{A})$. The first operator ${ }^{(0)}$ is supposed to be an infinitesimal generator that describes the behavior of the process between the jumps generated by the second operator $4^{(j u m p)}$ given in $(2.1)$.

Diffusion processes with jumps have been considered in [Gikhman/Skorokhod-1973, ch.1, \$1]. In this case, the operator $4^{(0)}$ in (2.11) has the form of a second order partial differential operator

$\mathscr{A}^{(0)}(\varphi)(z)=\left\langle a(z) \nabla_{z}, \nabla_{z}\right\rangle(\varphi)(z)+\left\langle b(z), \nabla_{z}\right\rangle(\varphi)(z)$,

where the function $b$ is $\mathbb{R}^{d}$-valued and the values of the function $a$ are positively definite d $\otimes$-matrices. The set $\mathscr{D}(\mathbb{A})$ consists of all sufficiently smooth bounded functions $\varphi$ on $\mathbb{R}^{d}$.

The approximate (up to higher orders of $\Delta t$ ) pathwise behavior of the process is described as follows. Let $z\left(t_{0}\right)=z$, then

where

$$
\mathrm{z}\left(\mathrm{t}_{\mathrm{o}}+\Delta \mathrm{t}\right)=\Delta \mathrm{z}_{1}+\Delta \mathrm{z}_{2}+\Delta \mathrm{z}_{3}
$$

$\Delta z_{1}$ is the deterministic term $b(z) \Delta t$,

$\Delta \mathrm{z}_{2}$ is normally distributed with zero mean and the correlation matrix $a(z) \Delta t$,

and $\Delta z_{3}$ is a jump term with the distribution

$$
(1-\lambda(z) \Delta t) \delta_{z}(.)+\lambda(z) \Delta t \lambda(z)^{-1} q(z, .) \text {. }
$$

Some additional assumptions allow to describe even the exact 
pathwise behavior of the process.

Consider a stochastic differential equation with a jump component (cf. [Gikhman/Skorokhod-1982, ch.6, 1 ], or [Ikeda/ Watanabe-1981, ch.4,§9])

$$
d z(t)=b(z(t)) d t+\sigma(z(t)) d W(t)+\int_{\Xi} f(z(t-), \xi) p(d \xi, d t) .
$$

The function $\sigma$ in $(2.13)$ is supposed to be d®d-matrix-valued and such that $a=\frac{1}{2} \sigma \sigma^{*}$, where $\sigma^{*}$ denotes the transposed matrix. Furthermore, $W$ denotes the Wiener process in the $\mathbb{R}^{d}$ and $p$ a random Poisson measure with an intensity measure $\pi(d \xi) d t$, where $\pi$ is a finite measure on the parameter set $\Xi$. Finally, the function $f$ on $\mathbb{R}^{d} \otimes E$ is $\mathbb{R}^{d}$-valued.

The solution process $Z$ of (2.13) has an infinitesimal generator of the form

$$
\not \mathbb{A}^{(0)}(\varphi)(z)+\int_{\Xi}[\varphi(z+f(z, \xi))-\varphi(z)] \pi(d \xi),
$$

where $4^{(0)}$ is given in (2.12).

Consequently, if the jump generator (2.1) has the special form (2.6) and the assumptions (2.7), (2.8), (2.10) are satisfied, then the corresponding diffusion process with jumps can be described via a stochastic differential equation of the form (2.13). The parameters are to be chosen in such a way that the second term in $(2.14)$ coincides with $(2.9)$, i.e.

$\Xi=[0,1], \xi=(\theta, \eta), \pi(\mathrm{d} \xi)=Q_{\max }(\theta) \mu(\mathrm{d} \theta) \mathbb{I}_{[0,1]}(\eta) \mathrm{d} \eta$, $f(z, \xi)=T(z, \theta) I_{I}(z, \theta, \eta), I=\left\{(z, \theta, \eta): \eta<Q(z, \theta) / Q_{\max }(\theta)\right\}$.

Now, the exact pathwise behavior of the process follows from the stochastic differential equation (2.13). Starting at $z\left(t_{0}\right)=z$, the process behaves like a diffusion during a random time $\tau$. This waiting time is exponentially distributed with the parameter

$$
\pi(\Xi)=\int Q_{\max }(\theta) \mu(\mathrm{d} \theta)
$$

and independent of the trajectory. At the time $t=t_{0}+\tau$, the jump parameter $\xi$ is generated according to the probability measure $\pi(\Xi)^{-1} \pi(d \xi)$, and the new state is calculated as 


$$
Z(t)=Z(t-)+f(Z(t-), \xi) \text {. }
$$

The interpretation of the fictitious jumps remains the previous one.

Piecewise deterministic processes have been investigated in [Davis-1984]. In this case, the operator $4^{(0)}$ in $(2.11)$ describes a deterministic process, including the pure drift case ((2.12) with $a=0)$ but also processes in bounded domains. The following pathwise behavior of such processes has been established. Starting at $z\left(t_{0}\right)=z$, the deterministic evolution goes on during a random waiting time $\tau$, which has the distribution

$$
\operatorname{Prob}\{\tau s\}=\exp \left(-\int_{0}^{s} \lambda\left(z\left(t_{0}+u\right)\right) d u\right), s \geq 0,
$$

where $\lambda$ is defined in (2.2). At the time $t=t_{0}+\tau$, the process jumps to a state $y \in \mathbb{R}^{d}$, which is distributed according to the probability measure

$$
\lambda(\mathrm{z}(\mathrm{t}-))^{-1} \mathrm{q}(\mathrm{z}(\mathrm{t}-), \mathrm{dy})
$$

Then, the evolution goes on analogously.

Suppose the jump generator (2.1) has the special form (2.6) and the assumptions (2.7), (2.8) are satisfied. In accordance with the two examples described above, the following conjecture concerning the pathwise behavior of a Markov process given by an infinitesimal generator of the form (2.11) seems to be reasonable.

Starting at $Z\left(t_{0}\right)=z$, the process behaves according to the infinitesimal generator ${ }^{(0)}$ during a random time $\tau$. This waiting time has the distribution

$$
\operatorname{Prob}\{\tau \geq s\}=\exp \left(-\int_{0}^{s} \lambda_{m a j}\left(z\left(t_{0}+u\right)\right) d u\right), s \geq 0 .
$$

At the time $t=t_{0}+\tau$, the jump parameter $\theta$ is generated according to the probability measure 


$$
\lambda_{\text {maj }}(Z(t-))^{-1} Q_{\text {maj }}(Z(t-), \theta) \mu(d \theta) \quad
$$

The generation of the parameter $\eta$ according to the uniform distribution on the unit interval corresponds to the decision whether a jump will take place or not. If

$$
\eta<Q(Z(t-), \theta) / Q_{m a j}(Z(t-), \theta),
$$

then the new state is calculated as

$$
Z(t)=Z(t-)+T(Z(t-), \theta) \text {. }
$$

Otherwise, $z(t)=Z(t-)$, i.e. the jump is fictitious.

Then, the evolution goes on analogously.

\section{THE basic Markov process}

In this section, we define a special Markov process $Z(t)$, $t \geq t_{0}$, with an infinitesimal generator of the form

$$
A=4^{(0)}+4^{(j u m p)} .
$$

The state space of the process is $\left(G \otimes \mathbb{R}^{3}\right)^{n}$, where $n$ is a natural number. We suppose the boundary $\partial G$ of the domain $G \subset \mathbb{R}^{3}$ to be sufficiently regular. Furthermore, we denote $z=\left(z_{i}\right), z \in\left(G \otimes \mathbb{R}^{3}\right)^{n}$, and $z_{i}=\left(x_{i}, v_{i}\right), i=1, \ldots n$.

This Markov process can be interpreted as a stochastic particle system

$$
\left(x_{i}(t), v_{i}(t)\right), i=1, \ldots, n \text {, }
$$

where $x_{i}(t)$ and $v_{i}(t)$ denote the position and the velocity of the $i-t h$ particle at the time $t$.

First we define the operator $4^{(0)}$, which describes the behavior of the process between the jumps (free flow of the particles).

The independence of different particles during the free flow corresponds to an operator of the form 


$$
\mathscr{A}^{(0)}(\Phi)(z)=\sum_{i=1}^{n} \mathbb{z}_{i}^{(\text {free })}(\Phi)(z),
$$

where $\Phi$ is an appropriate test function. The operator (free) is the infinitesimal generator governing the motion of a single particle during the free flow. We call this process the free flow process. The sign $\mathscr{A}_{i}^{(\mathrm{free})}$ denotes the operator $\mathbb{A}^{(\mathrm{free})}$ acting with respect to the components $z_{i}$.

In the interior of the domain $G$, the motion of a particle $(x(t), V(t))$ is described by the system of ordinary differential equations

$$
d X(t)=V(t) d t, \quad d V(t)=F(x(t), V(t)) d t,
$$

where $F$ denotes an external force acting on the particle.

It remains to define the behavior of the particle at the boundary $\partial G$. Suppose that the particle collides with the boundary at some time $t$. In this case, the position $x(t-)=x$ and the velocity $V(t-)=v$ of the particle satisfy the condition

$$
x \in \partial G \quad, \quad\langle v, n(x)\rangle \leq 0 \quad \text {. }
$$

During the collision, the velocity of the particle changes in such a way that the new velocity $v(t)=w$ satisfies the condition

$$
\langle w, n(x)\rangle>0
$$

We assume that $w$ is distributed according to a probability measure $r(x, v, d w)$ such that

$$
r(x, v,\{w:\langle w, n(x)>>0\})=1 \text {. }
$$

Then, the motion continues according to (3.3).

In order to calculate the infinitesimal generator of the free flow process, we consider a sufficiently smooth test function $\varphi$ on $G \otimes \mathbb{R}^{3}$.

Obviously,

$$
d^{(\text {free })}(\varphi)(x, v)=\left\langle v, \nabla_{x}\right\rangle(\varphi)(x, v)+\left\langle F(x, v), \nabla_{v}\right\rangle(\varphi)(x, v) \text {, }
$$

if $x \in G \backslash \partial G$ or if $x \in \partial G$ and $\langle v, n(x)>>0$, since the process is a 
pure drift process in these cases.

Suppose $x \in \partial G$ and $\langle v, n(x)\rangle \leq 0$. Then

$\mathbb{A}^{(f \text { ree })}(\varphi)(x, v)=\lim _{s \rightarrow 0} s^{-1}[E(x, v) \varphi(X(s), v(s))-\varphi(x, v)]$,

where the sign $E(x, v)$ denotes the expectation under the condition that $X(0)=x$ and $V(0)=v$. One obtains

$\mathbb{A}^{(f r e e)}(\varphi)(x, v)=\lim _{s \rightarrow 0} s^{-1}\left[\int_{\mathbb{R}^{3}} r(x, v, d w) E(x, w)^{\varphi(X(s), V(s))-\varphi(x, v)]}\right.$
$=\int_{\mathbb{R}^{3}} r(x, v, d w) d^{(f r e e)}(\varphi)(x, w)+\lim _{s \rightarrow 0} s^{-1}\left[\int_{\mathbb{R}^{3}} r(x, v, d w) \varphi(x, w)-\varphi(x, v)\right]$

according to $(3.4)$ and $(3.5)$. Consequently, the function $\varphi$ is subject to the condition

$\varphi(x, v)=\int_{\mathbb{R}^{3}} r(x, v, d w) \varphi(x, w), x \in \partial G,<v, n(x)>s 0$.

This condition and the smoothness assumptions necessary in (3.5) characterize the set $\mathscr{D}\left(\mathscr{A}^{\text {(free) }}\right)$.

Now we define the operator $x^{(j u m p)}$, which describes the jump mechanism (collisions between particles).

We consider an infinitesimal generator of the form (2.1), $(2.6)$. Let the parameter set be of the form

$$
\theta=\{(i, j): i, j=1, \ldots, n\} \otimes S^{2}
$$

and the measure $\mu$ be the product of the counting measure with respect to $(i, j)$ and a measure $m$ on $s^{2}$. Consequently, the jump distribution $(2.6)$ is modified in the following way,

$q(z, d y)=\sum_{i, j=1}^{n} \int_{s^{2}} \delta+T(z, i, j, e)(d y) Q(z, i, j, e) m(d e)$.

In order to introduce fictitious jumps, we assume that

and

$$
Q(z, i, j, e) \quad S \quad Q_{m a j}(z, i, j, e)
$$


$\lambda_{m a j}(z):=\sum_{i, j=1}^{n} \int_{s^{2}} Q_{m a j}(z, i, j, e) m(d e) \leq$ const $<\infty, \forall z$

The infinitesimal generator takes the form (cf. (2.9))

$\begin{aligned} \Phi^{(j u m p)}(\Phi)(z)= & \sum_{i, j=1}^{n} \int_{S^{2}} \int_{0}^{1}\left[\Phi\left(z+T(z, i, j, e) \Psi_{I}(z, i, j, e, \eta)\right)\right. \\ & -\Phi(z)] d \eta Q_{m a j}(z, i, j, e) m(d e),\end{aligned}$

$I:=\left\{(z, i, j, e, \eta): \eta<Q(z, i, j, e) / Q_{\operatorname{maj}}(z, i, j, e)\right\}$,

for continuous bounded functions $\Phi$. We call the corresponding process the collision process.

The motivation for the special choice of the jump parameters is to introduce a specific interaction in the particle system, namely collisions between two particles.

In this connection, it is natural to suppose

$$
\begin{aligned}
& Q(z, i, i, e)=0, \quad Q_{m a j}(z, i, i, e)=0, \\
& T(z, i, i, e)=0,
\end{aligned}
$$

for $i=1, \ldots, n$, and

$Q(z, i, j, e)=K\left(z_{i}, z_{j}, e\right), Q_{m a j}(z, i, j, e)=K_{m a j}\left(z_{i}, z_{j}, e\right)$,

$T(z, i, j, e)_{k}=\left\{\begin{array}{cl}0 & , k \neq i, j \\ \Psi_{1}\left(z_{i}, z_{j}, e\right) & , k=i \\ \Psi_{2}\left(z_{i}, z_{j}, e\right) & , k=j\end{array}\right.$,

for $i \neq j$, where $K, K_{m a j}, \Psi_{1}$ and $\psi_{2}$ are appropriate functions.

For several considerations, it will be convenient to specify the functions $\psi_{1}$ and $\psi_{2}$ via the relations

$\psi_{1}\left(z_{1}, z_{2}, e\right)=\varepsilon_{1} \psi\left(z_{1}, z_{2}, e\right), \psi_{2}\left(z_{1}, z_{2}, e\right)=\varepsilon_{2} \psi\left(z_{2}, z_{1}, e\right)$,

where $\psi$ is the usual collision transformation of the particles 
defined as

$$
\psi\left(\left(x_{1}, v_{1}\right),\left(x_{2}, v_{2}\right), e\right)=\left(0, e<e, v_{2}-v_{1}>\right)
$$

The introduction of the parameters $\varepsilon_{1}$ and $\varepsilon_{2}$, which may take the values 0 or 1 only, allows to consider the usual symmetric $\left(\varepsilon_{1}=\varepsilon_{2}=1\right)$ as well as asymmetric $\left(\varepsilon_{1}=1, \varepsilon_{2}=0\right)$ collisions. It also should be mentioned that we consider pairs of parameters $i \neq j$ (instead of $i<j$ only) in order to avoid symmetry assumptions concerning the functions $\mathrm{K}, \psi_{1}$ and $\psi_{2}$.

Under the additional assumption

$$
K_{m a j}\left(z_{1}, z_{2}, e\right)=K_{\max }(e)
$$

the collision process can be expressed via the stochastic differential equation (cf. (2.13))

$d Z(t)=\int_{\Xi} T(Z(t-), i, j, e) \mathbb{I}_{I}(Z(t-), i, j, e, \eta) p(d \xi, d t)$,

where $I:=\left\{(z, i, j, e, \eta): \eta<K\left(z_{i}, z_{j}, e\right) / K_{\max }(e)\right\}$,

$$
\begin{aligned}
\Xi: & =\left\{(i, j, e, \eta): i, j=1, \ldots n, \quad e \in S^{2}, \eta \in[0,1]\right\}, \text { and } \\
\pi(d \xi): & =K_{\max }(e) m(d e) \mathbb{I}_{[0,1]}(\eta) d \eta .
\end{aligned}
$$

The assumption ( 3.12 ) concerning the transformation $T$ as well as some elementary properties of random Poisson measures allow to write down the stochastic differential equation (3.15) in the following component-wise form,

$$
\begin{aligned}
d z_{i}(t) & =\sum_{j: j \neq i} \int_{s^{2} \otimes[0,1]} f_{1}\left(z_{i}(t-), z_{j}(t-), e, \eta\right) p^{(i, j)}(d e, d \eta, d t) \\
& +\sum_{j: j \neq i} \int_{s^{2} \otimes[0,1]} f_{2}\left(z_{j}(t-), z_{i}(t-), e, \eta\right) p^{(j, i)}(d e, d \eta, d t),
\end{aligned}
$$

where

$$
f_{k}\left(z_{1}, z_{2}, e, \eta\right)=\psi_{k}\left(z_{1}, z_{2}, e\right) \mathbb{I}_{\left\{\eta<K\left(z_{1}, z_{2}, e\right) / K_{\max }(e)\right\}}\left(z_{1}, z_{2}, e\right),
$$


for $k=1,2$, and $p^{(i, j)}, i, j=1, \ldots, n$, are independent random Poisson measures with the intensity measure

$$
K_{\max }(e) m(d e) \mathbb{I}_{[0,1]}(\eta) d \eta d t
$$

Now we consider the combined process corresponding to the infinitesimal generator (3.1). According to the conjecture stated at the end of the previous section, the pathwise behavior of this process is the following.

starting at $Z\left(t_{0}\right)=z$, the process behaves according to the infinitesimal generator (3.2) during a random time $\tau$. Thus, the particles perform independent motions in correspondence with the infinitesimal generator $\mathbb{4}^{\text {(free) }}$ of the free flow process.

The waiting time $\tau$ has the distribution (cf. (2.15))

where

$$
\operatorname{Prob}\{\tau z s\}=\exp \left(-\int_{0}^{s} \lambda_{m a j}\left(z\left(t_{0}+u\right)\right) d u\right),
$$

$$
\begin{aligned}
& \lambda_{\text {maj }}(z)=\sum_{i \neq j} \int_{S^{2}} K_{m a j}\left(z_{i}, z_{j}, e\right) m(d e), \\
& \text { according to }(3.8),(3.9), \text { and }(3.11) .
\end{aligned}
$$

At the $t i m e ~ t=t_{0}+\tau$, the jump parameter $\theta=(i, j, e)$ is generated according to the probability measure (cf. (2.16), $(3.9)$, and $(3.11))$

$$
\lambda_{m a j}(z(t-))^{-1} K_{m a j}\left(z_{i}(t-), z_{j}(t-), e\right) m(d e)
$$

Consequently, first the numbers $i$ and $j$ are calculated (choice of the collision partners) with respect to the probabilities

$$
\lambda_{m a j}(z(t-))^{-1} \int_{S^{2}} K_{m a j}\left(Z_{i}(t-), z_{j}(t-), e\right) m(d e)
$$

Given $i$ and $j$, the parameter $e$ is generated with respect to the probability measure 


$$
K_{m a j}\left(z_{i}(t-), z_{j}(t-), e\right) m(d e) / \int_{S^{2}} K_{m a j}\left(z_{i}(t-), z_{j}(t-), e\right) m(d e)
$$

The parameter $\eta$ is independent of the other parameters and has the uniform distribution on the interval $[0,1]$. The calculation of $\eta$ corresponds to the decision whether the collision is declared fictitious or not (cf. (2.17), (2.18), $(3,11)$ and $(3,12))$. If

$$
\eta \geq K\left(Z_{i}(t-), Z_{j}(t-), e\right) / K_{m a j}\left(Z_{i}(t-), Z_{j}(t-), e\right)
$$

i.e. with the probability

$$
1-K\left(Z_{i}(t-), Z_{j}(t-), e\right) / K_{\operatorname{maj}}\left(z_{i}(t-), Z_{j}(t-), e\right),
$$

the new state is $z(t)=Z(t-)$, i.e. no collision takes place or the collision is fictitious. Otherwise, i.e. with the probability

$$
K\left(Z_{i}(t-), Z_{j}(t-), e\right) / K_{m a j}\left(z_{i}(t-), z_{j}(t-), e\right)
$$

the new state is calculated as

$$
z_{k}(t)=z_{k}(t-)+\left\{\begin{array}{cl}
0 & , k \neq i, j \\
\psi_{1}\left(z_{i}(t-), z_{j}(t-), e\right) & , k=i \\
\psi_{2}\left(z_{i}(t-), z_{j}(t-), e\right) & , k=j
\end{array}\right.
$$

Thus, the components with the indices $i$ and $j$ are replaced by the corresponding "post-collision" values.

\section{ON THE ASYMPTOTIC BEHAVIOR OF THE EMPIRICAL MEASURES}

In this section, we study some properties of the basic Markov process $z(t), t \geq t_{0}$, with the infinitesimal generator

$$
4=4^{(0)}+4^{(j u m p)} .
$$

The operator ${ }^{(0)}$ is defined in (3.2) and the jump generator has the form 


$$
\mathbb{A}^{(j u m p)}(\Phi)(z)=\sum_{i, j=1}^{n} \int_{S^{2}}[\Phi(z+T(z, i, j, e))-\Phi(z)] K\left(z_{i}, z_{j}, e\right) m(d e),
$$

according to $(2.1),(3.7),(3.9)-(3.11)$. The transformation $T$ is given in $(3.12)$.

We are interested in the asymptotic behavior for large particle numbers $n$ of the empirical measure $v(t)$ associated with the process $z(t)$. This measure is defined via the relation $(\varphi, \nu(t)):=\int_{G \otimes R^{3}} \varphi(x, v) \nu(t, d x, d v):=n^{-1} \sum_{i=1}^{n} \varphi\left(z_{i}(t)\right)$,

for bounded measurable functions $\varphi$ on $G \otimes \mathbb{R}^{3}$.

The following representation is valid for general Markov processes (cf. [Skorokhod-1983, p.3], or [Davis-1984, p.26])

$\Phi(z(t))=\Phi\left(Z\left(t_{0}\right)\right)+\int_{t_{0}}^{t}(\Phi)(z(s)) d s+M(t)$,

where $M(t)$ is a martingale and $\Phi \in D(\mathcal{D})$.

We want to apply $(4.4)$ to the function

$$
\Phi(z)=n^{-1} \sum_{i=1}^{n} \varphi\left(z_{i}\right)
$$

This function belongs to $\mathscr{D}(\mathbb{A})$ if $\varphi$ is a sufficiently smooth bounded function satisfying condition (3.6). Notice that

$$
\Phi(z(t))=(\varphi, \nu(t))
$$

It follows from (3.2) that

$$
A^{(0)}(\Phi)(z)=n^{-1} \sum_{i=1}^{n} \phi^{(f r e e)}(\varphi)\left(z_{i}\right)
$$

According to $(4.3)$, one obtains

$$
\phi^{(0)}(\Phi)(Z(s))=\left(\phi^{(\text {free })}(\varphi), \nu(s)\right)
$$


Using $(4.2),(3.10)$ and $(3.12)$, we calculate

$$
\begin{aligned}
& A^{(j u m p)}(\Phi)(z)= \\
& n^{-1} \sum_{i \neq j} \int_{S^{2}} \sum_{k=1}^{n}\left[\varphi\left(z_{k}+T(z, i, j, e){ }_{k}\right)-\varphi\left(z_{k}\right)\right] K\left(z_{i}, z_{j}, e\right) m(d e)= \\
& n^{-1} \sum_{i \neq j} \int_{S^{2}}\left[\varphi\left(z_{i}+\psi_{1}\left(z_{i}, z_{j}, e\right)\right)-\varphi\left(z_{i}\right)+\right. \\
& \left.\varphi\left(z_{j}+\Psi_{2}\left(z_{i}, z_{j}, e\right)\right)-\varphi\left(z_{j}\right)\right] K\left(z_{i}, z_{j}, e\right) m(d e) .
\end{aligned}
$$

Consequently, according to $(4.3)$, one obtains

$$
\begin{aligned}
& A^{(j u m p)}(\Phi)(z(s))=n \int_{G \otimes \mathbb{R}^{3}} \int_{G \otimes \mathbb{R}^{3}} \int_{S^{2}}\left[\varphi\left(z_{1}+\psi_{1}\left(z_{1}, z_{2}, e\right)\right)-\varphi\left(z_{1}\right)+\right. \\
& \left.+\varphi\left(z_{2}+\psi_{2}\left(z_{1}, z_{2}, e\right)\right)-\varphi\left(z_{2}\right)\right] K\left(z_{1}, z_{2}, e\right) m(d e) \nu\left(s, d z_{1}\right) \nu\left(s, d z_{2}\right) \\
& -\int_{G \otimes \mathbb{R}^{3}} \int_{S^{2}}\left[\varphi\left(z+\psi_{1}(z, z, e)\right)-\varphi(z)+\right. \\
& \left.\varphi\left(z+\psi_{2}(z, z, e)\right)-\varphi(z)\right] K(z, z, e) m(d e) \nu(s, d z) .
\end{aligned}
$$

According to $(4.6)$ and $(4.7), \mathrm{Eq} \cdot(4.4)$ takes the form

$$
\begin{aligned}
(\varphi, \nu(t)) & =\left(\varphi, \nu\left(t_{0}\right)\right)+\int_{t_{0}}^{t}\left(\mathbb{A}^{(r r e \theta)}(\varphi), \nu(s)\right) d s+ \\
& +\int_{t_{0}}^{t} d^{(j u m p)}(\Phi)(Z(s)) d s+M(t),
\end{aligned}
$$

where $4^{(j u m p)}(\Phi)(Z(s))$ is given in $(4.8)$.

Notice that the measure $v$ as well as the martingale $M$ depend on $n$ (cf. (4.3), (4.4)). Furthermore, let the function $\mathrm{K}$ depend on $\mathrm{n}$.

Suppose that the following relations are fulfilled as $n \rightarrow \infty$, 


$$
\begin{aligned}
& v^{(n)}(t) \rightarrow P(t), \\
& M^{(n)}(t) \rightarrow 0, \quad \forall t \geq t_{0}, \\
& \quad \forall t \geq t_{0},
\end{aligned}
$$

and

$n K^{(n)}\left(z_{1}, z_{2}, e\right) \rightarrow H\left(z_{1}, z_{2}, e\right), \forall z_{1}, z_{2}, e$,

where $P$ and $H$ are appropriate functions. Under certain assumptions concerning the convergence in $(4.10)-(4.12)$, one can conclude from Eq.(4.9) that the limit $P(t)$ satisfies the equation,

$$
\begin{aligned}
(\varphi, P(t)) & =\left(\varphi, P\left(t_{0}\right)\right)+\int_{t_{0}}^{t}\left(A^{(\text {ree })}(\varphi), P(s)\right) d s+ \\
& +\int_{t_{0}}^{t} \int_{G \otimes \mathbb{R}^{3}} \int_{G \otimes \mathbb{R}^{3}} \int_{S^{2}}\left[\varphi\left(z_{1}+\psi_{1}\left(z_{1}, z_{2}, e\right)\right)-\varphi\left(z_{1}\right)+\right. \\
& \left.+\varphi\left(z_{2}+\psi_{2}\left(z_{1}, z_{2}, e\right)\right)-\varphi\left(z_{2}\right)\right] H\left(z_{1}, z_{2}, e\right) m(d e) P\left(s, d z_{1}\right) P\left(s, d z_{2}\right) d s .
\end{aligned}
$$

The equivalent differential form with respect to $t$ is

$$
\begin{aligned}
& \frac{d}{d t}(\varphi, P(t))=\left(\mathbb{A}^{(f \operatorname{ree})}(\varphi), P(t)\right)+ \\
& \quad+\int_{G \otimes R^{3}} \int_{G \otimes R^{3}} \int_{S^{2}}\left[\varphi\left(z_{1}+\psi_{1}\left(z_{1}, z_{2}, e\right)\right)-\varphi\left(z_{1}\right)+\right. \\
& \left.+\varphi\left(z_{2}+\psi_{2}\left(z_{1}, z_{2}, e\right)\right)-\varphi\left(z_{2}\right)\right] H\left(z_{1}, z_{2}, e\right) m(d e) P\left(t, d z_{1}\right) P\left(t, d z_{2}\right),
\end{aligned}
$$

with the initial condition

$$
P\left(t_{0}\right)=\lim _{n \rightarrow \infty} \nu^{(n)}\left(t_{0}\right)
$$

The function $\phi$ in $(4.13)$ is an arbitrary sufficiently smooth bounded test function that obeys condition ( 3.6$)$.

The various components that determine the basic Markov process (cf. $(4.1),(4.2))$ are present in Eq. (4.13), (4.14). 
The operator ${ }^{(\mathrm{free})}$ as well as the functions $\psi_{1}$ and $\psi_{2}$ appear explicitly, while the initial state $z\left(t_{0}\right)$ defines the empirical measure $v^{(n)}\left(t_{0}\right)$ appearing in (4.14) and the function $K$ defines the function $H$ via the relation (4.12).

According to (3.13), the second term on the right-hand side of Eq. (4.13) can be transformed in the following way,

$$
\begin{aligned}
& \int_{G \otimes R^{3}} \int_{G \otimes R^{3}} \int_{S^{2}}\left[\varphi\left(z_{1}+\varepsilon_{1} \psi\left(z_{1}, z_{2}, e\right)\right)-\varphi\left(z_{1}\right)+\varphi\left(z_{2}+\epsilon_{2} \psi\left(z_{2}, z_{1}, e\right)\right)-\right. \\
& \left.-\varphi\left(z_{2}\right)\right] H\left(z_{1}, z_{2}, e\right) m(d e) P\left(t, d z_{1}\right) P\left(t, d z_{2}\right)= \\
& \int_{G \otimes \mathbb{R}^{3}} \int_{G \otimes \mathbb{R}^{3}} \int_{S^{2}}\left[\epsilon_{1} \varphi\left(z_{1}+\psi\left(z_{1}, z_{2}, e\right)\right)-\varepsilon_{1} \varphi\left(z_{1}\right)+\varepsilon_{2} \varphi\left(z_{2}+\psi\left(z_{2}, z_{1}, e\right)\right)-\right. \\
& \left.-\varepsilon_{2} \varphi\left(z_{2}\right)\right] H\left(z_{1}, z_{2}, e\right) m(d e) P\left(t, d z_{1}\right) P\left(t, d z_{2}\right)= \\
& \int_{G \otimes \mathbb{R}^{3}} \int_{G \otimes \mathbb{R}^{3}} \int_{S^{2}}\left[\varphi\left(z_{1}+\psi\left(z_{1}, z_{2}, e\right)\right)-\varphi\left(z_{1}\right)\right] * \\
& *\left\{\varepsilon_{1} \mathrm{H}\left(\mathrm{z}_{1}, \mathrm{z}_{2}, e\right)+\varepsilon_{2} \mathrm{H}\left(\mathrm{z}_{2}, \mathrm{z}_{1}, \mathrm{e}\right)\right\} \mathrm{m}(\mathrm{de}) \mathrm{P}\left(\mathrm{t}, \mathrm{d} \mathrm{z}_{1}\right) \mathrm{P}\left(\mathrm{t}, \mathrm{dz} \mathrm{z}_{2}\right)
\end{aligned}
$$

Consequently, under the assumption (3.13), Eq.(4.13) takes the form

$$
\begin{aligned}
\frac{d}{d t}(\varphi, P(t))= & \left(\mathbb{A}^{(f r e)}(\varphi), P(t)\right)+ \\
\int_{G \otimes R^{3}} \int_{G \otimes \mathbb{R}^{3}} & \int S^{2}\left[\varphi\left(z_{1}+\psi\left(z_{1}, z_{2}, e\right)\right)-\varphi\left(z_{1}\right)\right] *
\end{aligned}
$$

$$
*_{H}^{(s y m)}\left(z_{1}, z_{2}, e\right) m(d e) P\left(t, d z_{1}\right) P\left(t, d z_{2}\right)
$$

The function

$H^{(8 y m)}\left(z_{1}, z_{2}, e\right)=\varepsilon_{1} H\left(z_{1}, z_{2}, e\right)+\epsilon_{2} H\left(z_{2}, z_{1}, e\right)$

is the symmetrization with respect to $z_{1}$ and $z_{2}$ of the function $H$ in the case of symmetric collisions $\left(\varepsilon_{1}=\varepsilon_{2}=1\right)$ and is identical with $H$ in the case of asymmetric collisions $\left(\varepsilon_{1}=1\right.$, $\varepsilon_{2}=0$ ). 


\section{Relation to the Boltzmann equation}

In this section, the relation of Eq.(4.15) to the Boltzmann equation will be examined.

We suppose that the measures $P(t)$ are absolutely continuous with respect to the Lebesgue measure. Let $p(t, x, v)$ denote the corresponding densities. In the following, we transform Eq. (4.15) in such a way that the test functions $\Phi$ may be excluded.

It follows from $(3.5)$ that $\left(\mathbb{A}^{(f \operatorname{rec})}(\varphi), P(t)\right)=$ $\int_{G} \int_{\mathbf{R}^{3}}\left(\left\langle v, \nabla_{x}\right\rangle(\varphi)(x, v)+\left\langle F(x, v), \nabla_{v}\right\rangle(\varphi)(x, v)\right) p(t, x, v) d v d x$

Applying the stokes formula

$$
\int_{G} \sum_{i=1}^{3} \frac{\partial}{\partial x_{i}} U_{i}(x) d x=-\int_{\partial G} \sum_{i=1}^{3} U_{i}(x) n_{i}(x) s(d x),
$$

where $U$ is a sufficiently smooth vector function and $s$ denotes the standard surface measure, we obtain the equality

$$
\begin{aligned}
& \int_{G}\left\langle v, \nabla_{x}\right\rangle(\varphi)(x, v) p(t, x, v) d x= \\
& \left.\left.=-\int_{G} \varphi(x, v)<v, \nabla_{x}\right\rangle_{p}(t, x, v) d x-\int_{\partial G} \varphi(x, v) p(t, x, v)<v, n(x)\right\rangle_{s}(d x) . \\
& \text { Furthermore, } \\
& \int_{\mathbb{R}^{3}}\left\langle F(x, v), \nabla_{v}\right\rangle(\varphi)(x, v) p(t, x, v) d v=-\int_{\mathbb{R}^{3}} \varphi(x, v)<F(x, v), \nabla_{v}>p(t, x, v) d v \\
& \text { so that one obtains from (5.1) the relation }
\end{aligned}
$$


$-\left(\alpha^{(r e e)}(\varphi), P(t)\right)=$

$\int_{G} \int_{\mathbb{R}^{3}} \varphi(x, v)\left(\left\langle v, \nabla_{x}>p(t, x, v)+\left\langle F(x, v), \nabla_{v}>p(t, x, v)\right) d v d x\right.\right.$

$$
+\int_{\partial G} \int_{R^{3}} \varphi(x, v) p(t, x, v)<v, n(x)>s(d x) d v
$$

In order to handle the second term on the right-hand side of Eq.(4.15), we introduce a transformation $s$ by the formulas

$$
\begin{aligned}
& S_{1}\left(z_{1}, z_{2}, e\right):=z_{1}+\psi\left(z_{1}, z_{2}, e\right) \\
& S_{2}\left(z_{1}, z_{2}, e\right):=z_{2}+\psi\left(z_{2}, z_{1}, e\right)
\end{aligned}
$$

where $\psi$ is defined in (3.14). Obviously, the transformation $S$ is linear in $\left(z_{1}, z_{2}\right)$. Furthermore, elementary calculations show that $S^{-1}=S$.

The substitution of the variables

$$
y_{1}=s_{1}\left(z_{1}, z_{2}, e\right) \quad, \quad y_{2}=s_{2}\left(z_{1}, z_{2}, e\right)
$$

allows to conclude that

$$
\begin{gathered}
\int_{G \otimes \mathbb{R}^{3}} \int_{G \otimes \mathbb{R}^{3}} \varphi\left(z_{1}+\psi\left(z_{1}, z_{2}, e\right)\right) H^{(s y m)}\left(z_{1}, z_{2}, e\right) p\left(t, z_{1}\right) p\left(t, z_{2}\right) d z_{1} d z_{2}= \\
\int_{G \otimes \mathbb{R}^{3}} \int_{G \otimes R^{3}} \varphi\left(z_{1}\right) H^{(8 y m)}\left(S_{1}\left(z_{1}, z_{2}, e\right), S_{2}\left(z_{1}, z_{2}, e\right), e\right) * \\
p\left(t, S_{1}\left(z_{1}, z_{2}, e\right)\right) p\left(t, S_{2}\left(z_{1}, z_{2}, e\right)\right) d z_{1} d z_{2} .
\end{gathered}
$$

Consequently, the second term on the right-hand side of Eq.(4.15) takes the form

$$
\begin{aligned}
& \int_{G \otimes R^{3}} \varphi\left(z_{1}\right) \int_{G \otimes R^{3}} \int_{S^{2}}\left\{H^{(s y m)}\left(S_{1}\left(z_{1}, z_{2}, e\right), S_{2}\left(z_{1}, z_{2}, e\right), e\right) *\right. \\
& * p\left(t, S_{1}\left(z_{1}, z_{2}, e\right)\right) p\left(t, S_{2}\left(z_{1}, z_{2}, e\right)\right) \\
&\left.-H^{(s y m)}\left(z_{1}, z_{2}, e\right) p\left(t, z_{1}\right) p\left(t, z_{2}\right)\right\} m(d e) d z_{2} d z_{1} .
\end{aligned}
$$


Thus, according to (5.2) and (5.4), Eq.(4.15) can be transformed into the equation

$$
\begin{aligned}
& \int_{G} \int_{\mathbb{R}^{3}} \varphi(x, v)\left[\frac{\partial}{\partial t}+\left\langle v, \nabla_{x}\right\rangle+\left\langle F(x, v), \nabla_{v}\right\rangle\right] p(t, x, v) d v d x \\
& +\int_{\partial G} \int_{\mathbb{R}^{3}} \varphi(x, v) p(t, x, v)<v, n(x)>\operatorname{dvs}(d x)= \\
& \int_{G \otimes \mathbb{R}^{3}} \varphi\left(z_{1}\right) \int_{G \otimes R^{3}} \int_{S^{2}}\left\{H^{(8 y m)}\left(S_{1}\left(z_{1}, z_{2}, e\right), S_{2}\left(z_{1}, z_{2}, e\right), e\right) *\right. \\
& * p\left(t, s_{1}\left(z_{1}, z_{2}, e\right)\right) p\left(t, s_{2}\left(z_{1}, z_{2}, e\right)\right) \\
& \left.-H^{(s y m)}\left(z_{1}, z_{2}, e\right) p\left(t, z_{1}\right) p\left(t, z_{2}\right)\right\} m(d e) d z_{2} d z_{1} \text {, }
\end{aligned}
$$

where $\varphi$ is an arbitrary sufficiently smooth bounded test function satisfying condition $(3.6)$.

We notice that Eq. $(5.5)$ is fulfilled, in particular, for all sufficiently smooth bounded functions $\varphi$ such that

$$
\varphi(x, v)=0 \text {, if } x \in \partial G
$$

If a function $\varphi$ satisfies condition (5.6), then the second term on the left-hand side of Eq.(5.5) disappears. Furthermore, the class of functions satisfying (5.6) is sufficiently wide in order to conclude from Eq.(5.5) that the density $p(t, x, v)$ satisfies the equation

$$
\begin{aligned}
& {\left[\frac{\partial}{\partial t}+\left\langle v, \nabla_{x}\right\rangle+\left\langle F(x, v), \nabla_{v}\right\rangle\right] p(t, x, v)=} \\
& \iint_{G} \int_{S^{2}}\left\{H^{(s y m)}\left(x, v+e\left\langle e, v_{1}-v\right\rangle, x_{1}, v_{1}+e<e, v-v_{1}\right\rangle, e\right) * \\
& \left.\left.* p\left(t, x, v+e<e, v_{1}-v\right\rangle\right) p\left(t, x_{1}, v_{1}+e<e, v-v_{1}\right\rangle\right) \\
& \left.-H^{(s y m)}\left(x, v, x_{1}, v_{1}, e\right) p(t, x, v) p\left(t, x_{1}, v_{1}\right)\right\} m(d e) d v_{1} d x_{1},
\end{aligned}
$$


for all $x \in G \backslash \partial G, v \in \mathbb{R}^{3}$, and $t>t_{0}$.

As a consequence of Eq.(5.7), a second equation with respect to the densities $p(t, x, v)$ can be derived from Eq. (5.5). Namely, according to $(5.7)$, Eq. $(5.5)$ reduces to the equation

$$
\int_{\partial G} \int_{\mathbb{R}^{3}} \varphi(x, v) p(t, x, v)<v, n(x)>d v s(d x)=0
$$

which is fulfilled for arbitrary sufficiently smooth bounded test functions $\varphi$ satisfying condition (3.6).

For any fixed $x \in \partial G$, we introduce the half-spaces

$$
\mathbb{R}_{+}^{3}(x):=\left\{v \in \mathbb{R}^{3}:\langle v, n(x)>>0\} \text { and } \mathbb{R}_{-}^{3}(x):=\left\{v \in \mathbb{R}^{3}:\langle v, n(x)>\leq 0\}\right.\right.
$$

We suppose the measure $r$ to be absolutely continuous with respect to the Lebesgue measure and denote the corresponding density by $R$ so that the condition (3.6) takes the form

$\varphi(x, v)=\int_{\mathbb{R}^{3}} R(x, v, w) \varphi(x, w) d w, x \in \partial G,\langle v, n(x)>\leq 0$

Remember that, according to the assumption (3.4), the measure $r$ is concentrated on the half-space $\mathbb{R}_{+}^{3}(x)$.

Using $(5.9)$, one obtains the equality

$$
\begin{aligned}
& \int_{\mathbb{R}^{3}} \varphi(x, v) p(t, x, v)<v, n(x)>d v=\int_{\mathbb{R}_{+}^{3}(x)} \varphi(x, v) p(t, x, v)<v, n(x)>d v+ \\
& +\int_{-}^{3}(x)\left[\int_{+}^{3}(x)\right. \\
& =\int_{\mathbb{R}_{+}^{3}(x)} \mathrm{dv} \varphi(x, v)\{p(t, x, v)\langle v, n(x)\rangle+ \\
& \left.\int_{-}^{3}(x) R(x, w, v) p(t, x, w)<w, n(x)>d w\right\}
\end{aligned}
$$

for any fixed $x \in \partial G$. Consequently, it follows from Eq.(5.8) that 


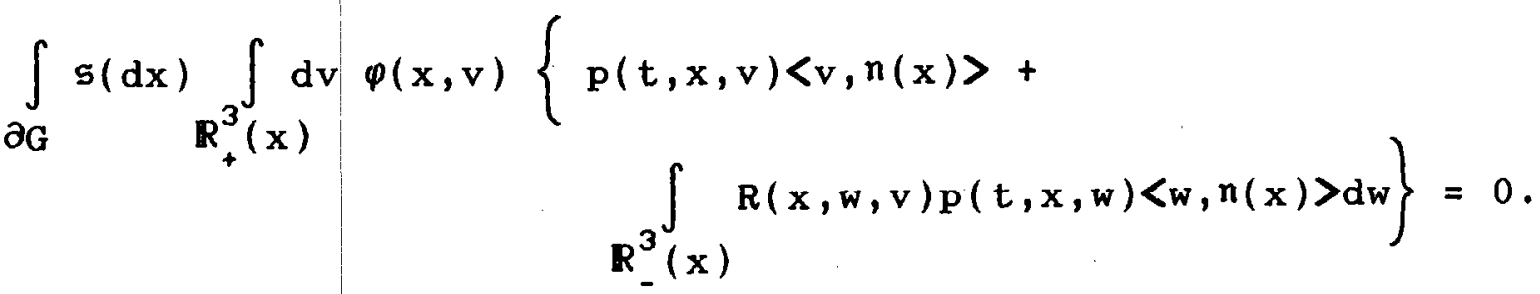

The class of test functions $\varphi$ is sufficiently wide in order to conclude that the function $p$ satisfies the equation

$p(t, x, v)\left\langle v, n(x)>+\int_{\mathbb{R}_{-}^{3}(x)} R(x, w, v) p(t, x, w)<w, n(x)>d w=0\right.$,

for any $x \in \partial G$ and $v \in R_{+}^{3}(x)$.

Thus, the function $p(t, x, v)$ satisfies Eq.(5.7) in the interior of the domain G, and Eq.(5.10) on the boundary $\partial G$. In fact, these two equations are equivalent to Eq.(4.15). The initial condition $(4.14)$ is to be modified appropriately.

The function $\mathrm{H}^{(8 \mathrm{ym})}$ that appears in Eq. (5.7) is determined by means of the parameter $K^{(n)}$ of the stochastic particle system (cf. $(4.2),(4.12)$, and $(4.16))$.

Let the parameter $K^{(n)}$ be of the special form

$$
\mathrm{K}^{(n)}\left(\mathrm{x}_{1}, \mathrm{v}_{1}, \mathrm{x}_{2}, \mathrm{v}_{2}, e\right)=n^{-1} k^{(n)}\left(\mathrm{x}_{1}, \mathrm{x}_{2}\right) \ell\left(\mathrm{v}_{1}, \mathrm{v}_{2}, e\right)
$$

where $k^{(n)}$ and $b$ are appropriate functions, and suppose that

$$
k^{(n)}\left(x_{1}, x_{2}\right) \underset{n \rightarrow \infty}{\rightarrow} \vartheta\left(x_{1}-x_{2}\right)
$$

Then, one obtains the relations

and

$$
\mathrm{H}\left(\mathrm{x}_{1}, \mathrm{v}_{1}, \mathrm{x}_{2}, \mathrm{v}_{2}, \mathrm{e}\right)=\vartheta\left(\mathrm{x}_{1}-\mathrm{x}_{2}\right) \&\left(\mathrm{v}_{1}, \mathrm{v}_{2}, \mathrm{e}\right),
$$

$$
\mathrm{H}^{(8 \mathrm{ym})}\left(\mathrm{x}_{1}, \mathrm{v}_{1}, \mathrm{x}_{2}, \mathrm{v}_{2}, e\right)=\vartheta\left(\mathrm{x}_{1}-\mathrm{x}_{2}\right) \mathrm{B}\left(\mathrm{v}_{1}, \mathrm{v}_{2}, e\right) \text {, }
$$

where

$$
\mathrm{B}\left(\mathrm{v}_{1}, \mathrm{v}_{2}, \mathrm{e}\right)=\varepsilon_{1} b\left(\mathrm{v}_{1}, \mathrm{v}_{2}, \mathrm{e}\right)+\varepsilon_{2} b\left(\mathrm{v}_{2}, \mathrm{v}_{1}, \mathrm{e}\right) .
$$


Suppose, furthermore, that the function $B$ satisfies the condition

$$
\mathrm{B}\left(\mathrm{v}^{*}, \mathrm{v}_{1}^{*}, \mathrm{e}\right)=\mathrm{B}\left(\mathrm{v}, \mathrm{v}_{1}, \mathrm{e}\right)
$$

where $v^{*}=v+e\left\langle e, v_{1}-v\right\rangle$ and $v_{1}^{*}=v_{1}+e\left\langle e, v-v_{1}\right\rangle$.

Under the assumptions (5.11), (5.12) and (5.14), Eq.(5.7) reduces to the Boltzmann equation

$\frac{\partial}{\partial t} p(t, x, v)+\left\langle v, \nabla_{x}\right\rangle_{p}(t, x, v)+\left\langle F(x, v), \nabla_{v}\right\rangle_{p}(t, x, v)=$ $\int_{\mathbb{R}^{3}} d v_{1} \int_{S^{2}} m(d e) B\left(v, v_{1}, e\right)\left\{p\left(t, x, v^{*}\right) p\left(t, x, v_{1}^{*}\right)-p(t, x, v) p\left(t, x, v_{1}\right)\right\}$.

Formula (5.13) establishes the relation between the parameter $b$ of the stochastic particle system and the collision kernel $B$ of the limiting Boltzmann equation.

If the collisions in the stochastic particle system are symmetric $\left(\varepsilon_{1}=\varepsilon_{2}=1\right)$, then the kernel $B$ is automatically a symmetric function with respect to $v_{1}$ and $v_{2}$. Consequently, one can choose the symmetric parameter

$$
b\left(v_{1}, v_{2}, e\right)=B\left(v_{1}, v_{2}, e\right) / 2
$$

In this case, it is also possible to reduce the possible collision partners in the stochastic model to ordered pairs $(i<j)$ and to choose $b=B$.

If the collisions in the stochastic particle system are asymmetric $\left(\varepsilon_{1}=1, \varepsilon_{2}=0\right)$, then the kernel $B$ and the parameter $b$ are identical.

It is simple to establish that

$$
\left\|v^{*}-v_{1}^{*}\right\|=\left\|v-v_{1}\right\| \text { and }\left\langle v^{*}-v_{1}^{*}, e\right\rangle=-\left\langle v-v_{1}, e\right\rangle \text {. }
$$

Consequently, condition (5.14) is fulfilled if $B$ depends on $v$ and $v_{1}$ via the terms $\left\|v-v_{1}\right\|$ and $\left|\left\langle v-v_{1}, e\right\rangle\right|$ only. This is fulfilled for many concrete collision kernels B. Obviously, the 
function $B$ is symmetric in this case.

Condition $(5.10)$ is one of the common boundary conditions associated with, the Boltzmann equation (cf. [Cercignani-1975, Ch.3, \$1]. If the kernel $R$ has the form

$$
R(x, w, v)=(1-\alpha) \vartheta\left(w-v+2 n(x)\langle n(x), v>)+\alpha f_{0}(x, v),\right.
$$

where $f_{0}$ is an appropriate density with respect to $v$ and $\alpha \in[0,1]$, one obtains the Maxwell boundary condition. In the case of pure specular reflection $(\alpha=0)$, equation (5.10) reduces to the condition

$$
p(t, x, v)=p(t, x, v-2 n(x)\langle n(x), v\rangle)
$$

Finally, it should be mentioned that, if one replaces the condition ( 5.12 ). by the following,

$$
k^{(n)}\left(x_{1}, x_{2}\right) \underset{n \rightarrow \infty}{\longrightarrow} h\left(x_{1}, x_{2}\right),
$$

where $h$ is an appropriate symmetric function, then Eq.(5.7) reduces to the so-called mollified Boltzmann equation (cf. [Lebowitz/Montroll-1983, p.34]).

\section{REFERENCES}

A.A.Arsen'ev, o priblizhenii resheniya uravneniya Bol'tsmana resheniyami stokhasticheskikh differentsial'nykh uravnenij Ito, Zh.Vychisl.Mat.i Mat.Fiz. 27, 3, 400-410 (1987).

H. Babovsky, On a simulation scheme for the Boltzmann equation, Math.Meth.Appl.Sci. 8, 223-233 (1986).

O.M.Belotserkovskij and V.E.Yanitskij, Statisticheskij metod chastits $v$ yachejkakh dlya resheniya zadach dinamiki razrezhennogo gaza. I. Osnovy postroeniya metoda, Zh.Vychisl. Mat.i Mat.Fiz. 15, 5, 1195-1209 (1975). 
C.Cercignani, Theory and Application of the Boltzmann Equation, Scottish Academic Press, Edinburgh, 1975.

C.Cercignani, The Boltzmann Equation and its Applications, Springer, New York, 1988.

M.H.A.Davis, Lectures on Stochastic Control and Nonlinear Filtering, Tata Institute of Fundamental Research, Bombay, 1984 .

S.M.Ermakov, V.V.Nekrutkin, and A.S.Sipin, Random Processes for Classical Equations of Mathematical Physics, Nauka, Moscow, 1984. (In Russian. English translation: Kluwer Academic Publishers, Dordrecht/Boston/London, 1989)

S.N.Ethier and T.G.Kurtz, Markov Processes, Characterization and Convergence, Wiley, New York, 1986.

W.Feller, An Introduction to Probability Theory and its Applications. Vol.2, Wiley, New York, 1966.

I.I.Gikhman and A.V.Skorokhod, Theory of Random Processes, Nauka, Moscow, 1971 (Vol.1), 1973 (Vol.2), 1975 (Vol.3). (In Russian )

I.I.Gikhman and A.V.Skorokhod, Stochastic Differential Equations and their Applications, Naukova Dumka, Kiev, 1982 . (In Russian)

F.Gropengiesser, H.Neunzert and J.Struckmeier, Computational methods for the Boltzmann equation, in: The state of Art in Applied and Industrial Mathematics (ed. R.Spigler), Kluwer Academic Publishers, Dordrecht, 1990.

N.Ikeda und S.Watanabe, Stochastic Differential Equations and Diffusion Processes, North-Holland, Amsterdam, 1981. 
R.Illner and H.Neunzert, On simulation methods for the Boltzmann equation, Transport Theory and Statistical Physics 16 (2\&3), 141-154 (1987).

M.S.Ivanov and S.V.Rogazinskij, Analysis of numerical techniques of the direct simulation Monte Carlo method in the rarefied gas dynamics, Sov.J.Numer.Anal.Math.Modelling 3, 6, 453-465 (1988).

M.S.Ivanov and S.V.Rogazinskij, Ehkonomichnye skhemy pryamogo statisticheskogo modelirovaniya techenij razrezhennogo gaza, Matem.Modelirovanie 1, 7, 130-145 (1989).

M.Kac, Foundations of kinetic theory, Proc.Third Berkeley Symp. Math.Stat.Prob. 3, 171-197 (1956).

M.Kac, Probability and Related Topics in the Physical Sciences, Interscience, New York, 1959.

A.I.Khisamutdinov, Ob imitatsionnom metode statisticheskogo modelirovaniya razrezhennykh gazov, Dokl.Akad.Nauk SSSR 291, 6, 1300-1303 (1986).

Yu.N.Kondyurin, Ob odnom statisticheskom podkhode $k$ resheniyu uravneniya Bol'tsmana, Zh.Vychisl.Mat.i Mat.Fiz. 26, 10, 1527$1534(1986)$.

J.L.Lebowitz and E.W.Montroll (eds.), Nonequilibrium Phenomena. I. The Boltzmann Equation, North-Holland, Amsterdam, 1983.

M.A.Leontovich, Osnovnye uravneniya kineticheskoj teorii gazov s tochki zreniya teorii sluchajnykh protsessov, Zhurnal Teoret. Ehksper. Fiziki 5, 211-231 (1935).

A.V.Lukshin, Stokhasticheskie algoritmy matematicheskoj teorii prostranstvenno-neodnorodnogo uravneniya Bol'tsmana, Matem. Modelirovanie 1, 7, 146-159 (1989). 
H.P.McKean, Speed of approach to equilibrium for Kac's caricature of a Maxwellian gas, Arch.Rat.Mech.Anal. 21, 343-367 $(1966)$.

H.P.Mckean, Fluctuations in the kinetic theory of gases, Comm. Pure Appl.Math. 28, 4, 435-455 (1975).

K.Nanbu, Interrelations between various direct simulation methods for solving the Boltzmann equation, J.Phys.Soc.Jpn. 52, $10,3382-3388(1983)$.

Proceedings of the 16 th International Symposium on Rarefied Gas Dynamics (Eds. E.P.Muntz, D.Weaver, D.Campbell), Progress in Astronautics and Aeronautics, vols. 116-118, AIAA, New York, 1989 .

A.V.Skorokhod, Stochastic Equations for Complex Systems, Nauka, Moscow, 1983. (In Russian. English translation: Reidel, Dordrecht, 1988)

S.N.Smirnov, $K$ obosnovaniyu odnogo stokhasticheskogo metoda resheniya uravneniya Bol'tsmana, Zh.Vychisl.Mat.i Mat.Fiz. 29, $2,270-276(1989)$.

A.D.Venttsel', Lectures on the Theory of Random Processes, Nauka, Moscow, 1975. (In Russian) 